\title{
Epigenetic Mechanisms Underlying Organic Solute Transporter $\beta$ Repression in Colorectal Cancer ${ }^{\mathbb{8}}$
}

\author{
Ying Zhou, ${ }^{1}$ Chaonan Ye, ${ }^{1}$ Yan Lou, ${ }^{2}$ Junqing Liu, Sheng Ye, Lu Chen, Jinxiu Lei, \\ Suhang Guo, Su Zeng, and Lushan Yu
}

Institute of Drug Metabolism and Pharmaceutical Analysis, College of Pharmaceutical Sciences, Zhejiang University, Hangzhou, Zhejiang, China (Y.Z., C.Y., L.C., J.Le., S.G., S.Z., L.Y.); Departments of Pharmacy (Y.L.) and Radiation Oncology (J.Li.), The First Affiliated Hospital and Intensive Care Unit, The Children's Hospital (S.Y.), School of Medicine, Zhejiang University, Hangzhou, Zhejiang, China

Received September 1, 2019; accepted January 24, 2020

\begin{abstract}
Colorectal cancer (CRC) is known to be the third most common cancer disease and the fourth-leading cause of cancer-related deaths worldwide. Bile acid, especially deoxycholic acid and lithocholic acid, were revealed to play an important role during carcinogenesis of CRC. In this study, we found organic solute transporter $\beta$ (OST $\beta)$, an important subunit of a bile acid export transporter OST $\alpha$-OST $\beta$, was noticeably downregulated in CRC. The decline of OST $\beta$ expression in CRC was determined by Western blot and real-time polymerase chain reaction (RTPCR), whereas chromatin immunoprecipitation (ChIP) was used to evaluate the histone acetylation state at the OST $\beta$ promoter region in vivo and in vitro. CRC cell lines HT29 and HCT15 were treated with trichostation A (TSA) for the subsequent determination, including RT-PCR, small interfering RNA (siRNA) knockdown, ChIP, and dual-luciferase reporter gene assay, to find out which histone acetyltransferases and deacetylases exactly participated in regulation. We demonstrated that after TSA treatment, OST $\beta$ expression increased noticeably because of upregulated H3K27Ac state at OST $\beta$
\end{abstract}

promoter region. We found that stimulating the expression of p300 with CTB (Cholera Toxin B subunit, an activator of p300) and inhibiting p300 expression with C646 (an inhibitor of p300) or siRNA designed for $p 300$ could control OST $\beta$ expression through modulating H3K27Ac state at OST $\beta$ promoter region. Therefore, downregulated expression of p300 in CRC may cause low expression of OST $\beta$ in CRC via epigenetic regulation. Generally, we revealed a novel epigenetic mechanism underlying OST $\beta$ repression in CRC, hoping this mechanism would help us to understand and inhibit carcinogenesis of $\mathrm{CRC}$.

\section{SIGNIFICANCE STATEMENT}

Organic solute transporter $\beta$ (OST $\beta$ ) expression is lower in colon cancer tissues compared with adjacent normal tissues. We revealed the epigenetic mechanisms of it and proved that p300 controls OST $\beta$ expression through modulating H3K27Ac state at OST $\beta$ promoter region and hence causes low expression of OST $\beta$ in colorectal cancer.

\section{Introduction}

Colorectal cancer (CRC) is known to be the third most common cancer disease and the fourth-leading cause of cancer-related deaths worldwide (Favoriti et al., 2016). CRC rate is geographically relevant; North America, Oceania, and Europe have higher incidence of CRC, whereas Asia, Africa, and South America show the lowest CRC incidence rates. Such phenomenon may be connected to risk factors, such as obesity and physical inactivity (Center et al., 2009). Although surgery intervention and neoadjuvant and adjuvant chemotherapy were the

This work was supported by the grants from the National Natural Science Foundation of China [81872935 and 81773805] and the Fundamental Research Funds for the Central Universities [2017XZZX011-04].

${ }^{1}$ Y.Z. and C.Y. contributed equally to this work.

${ }^{2}$ Y.L. contributed equally to this work.

https://doi.org/10.1124/mol.119.118216.

S This article has supplemental material available at molpharm. aspetjournals.org. most common and efficient methods in CRC treatment (Brenner et al., 2014), early diagnosis and useful therapeutic targets in CRC are becoming more and more important to assist therapy.

During carcinogenesis and development of CRC, bile acids seem to play a vital role. Bile acids are physiologic products synthesized from cholesterol in liver and functioned as generating bile flow as well as promoting intestinal absorption and transportation of lipids, nutrients, and vitamins (Chiang, 2009). Narisawa et al. reveals lithocholic acid (LCA) and deoxycholic acid (DCA) promote CRC in rats after MNNG ( $N$-methyl- $N$ '-nitro- $N$-nitrosoguanidine)treatment (Narisawa et al., 1974). Bernstein et al. further confirms the effect of DCA in a mouse experiment; they fed 18 mice a $0.2 \%$ DCAcontaining diet, and after 8-10 months, 10 of them had CRC (Bernstein et al., 2011). Recently, more studies have found the secondary bile acids LCA and DCA are the main factors involved in carcinogenesis and development of CRC (Plotnikoff, 2014; Farhana et al., 2016).

ABBREVIATIONS: CRC, colorectal cancer; CTB, cholera toxin B subunit; DAC, decitabine; DCA, deoxycholic acid; GAPDH, glyceraldehyde-3phosphate dehydrogenase; HAT, histone acetyltransferase; HDAC, histone deacetylase; LCA, lithocholic acid; OST, organic solute transporter; PPIB, peptidylprolyl isomerase B; qRT-PCR, quantitative real-time polymerase chain reaction; siRNA, small interfering RNA; TSA, trichostation A. 
Organic solute transporter (OST) $\alpha$ and OST $\beta$ form a heteromeric transporter known as OST- $\alpha / \beta$, which participates in enterohepatic circulation of bile acids (Ballatori et al., 2013). The two subunits of the transporter are expressed together in human small intestine, kidney, liver, colon, testis, and ovary, with a high expression in inileum, as shown when searched in the Variability of Drug Transporter Database (Yin et al., 2020). They localize at basolateral plasma membrane of epithelial cells responsible for bile acids efflux (Seward et al., 2003; Ballatori et al., 2005). Although maintainment of the transport activity of this transporter complex requires the normal interaction between OST $\alpha$ and OST $\beta$ subunits, OST $\beta$ was reported to function as an accessory factor to promote the egress of OST $\alpha$ as well as stabilize the heterodimerization complex, traffic the complex to plasma membrane, and maintain the bile acid transport activity (Christian et al., 2012). Therefore, it is reasonable to speculate that abnormal OST $\beta$ expression can influence transport capability of OST $\alpha$-OST $\beta$ transporter, and the OST $\beta$ is the main object in our research.

Epigenetic alterations have attracted more attention in recent years and are considered to play a key role during CRC development (Okugawa et al., 2015). Epigenetics refers to the study of heritable alterations in gene expression without undergoing changes in DNA sequence, including DNA methylation, histone modification, noncoding RNA expression, and nucleosome remodeling (Bird, 2002; Liu et al., 2016). Among those, histone modifications include acetylation, methylation, phosphorylation, ubiquitylation, ADP-ribosylation, and deamination (Minucci and Pelicci, 2006), controlled by the involvements of some key enzymes such as histone acetyltransferases (HATs) and deacetylases (HDACs). Actually, histone acetylation is an indicator of active gene transcription because the hyperacetylation state of histones relaxes the chromatin structure with increasing transcriptional activity, whereas hypoacetylation state of histones condenses the chromatin structure with decreasing transcriptional activity (Codd et al., 2009). In other words, the two different enzymes HATs and HDACs can cause gene activation and silencing on the chromosome level, respectively, as well as control specific gene expression in vivo (Wang et al., 2009).

In this study, we found that OST $\beta$ expression was distinctly repressed in CRC tissues. To clarify which factors were involved, we determined regulatory mechanisms via epigenetic control that underlie this phenomenon.

\section{Materials and Methods}

Tissues and Cell Culture. A total of 93 paired tissue samples from CRC patients in this study were collected from the Specimen Bank of Zhejiang Cancer Hospital (Hangzhou, China), with the approval by the Institutional Review Board of Zhejiang Cancer Hospital. Detailed patient information is provided in Supplemental Table 1. All the experiments of patient tissues were in line with the Helsinki Declaration. Those samples were well-kept under $-80^{\circ} \mathrm{C}$ without specific classification. The reference gene (PPIB, peptidylprolyl isomerase B) values were first detected, and paired normal and cancer tissues that contained similar PPIB values were chosen for the subsequent analysis. Colon cancer cell lines HCT15 were gifted from the Institute of Modern Chinese Medicine, College of Pharmaceutical Sciences, Zhejiang University; HT29 cells were purchased from Cell Bank of the Chinese Academy of Sciences (Shanghai, China). Both cell lines were cultured in RPMI 1640 medium (Gibco) containing $10 \% \mathrm{FBS}, 100 \mathrm{U} / \mathrm{ml}$ penicillin, and $100 \mathrm{mg} / \mathrm{ml}$ streptomycin in a humidified atmosphere at $37^{\circ} \mathrm{C}$ with $5 \% \mathrm{CO}_{2}$, under the guidance of instructions from American Type Culture Collection. During the whole process of the experiment, cell growth situation and cell culture medium were checked every time before the cell operations, and nonexistence of mycoplasma contamination has been spotted.

Cell Drug Treatments. Decitabine (DAC), cholera toxin B subunit (CTB), and C646 were purchased from Sigma-Aldrich, and TSA was purchased from TCI. HCT115 cells were previously seeded in a six-well plate with $10 \%-20 \%$ cell density and cultured with medium containing different doses of DAC for 72 hours, with refreshing culture medium every day. For CTB treatment, cells were precultured in 40\%$50 \%$ confluence, and after the overnight culture, cells were treated with medium containing indicated doses of CTB for another 48 hours. The medium was refreshed every day. For TSA and C646 treatment, HCT15 was precultured to $30 \%$ confluence and HT29 was precultured to $60 \%$ confluence in a six-well plate, and then cells were subsequently cultured with medium containing indicated doses of TSA and C646 for 24 hours.

RNA Extraction and Real-Time Quantitative Polymerase Chain Reaction Analysis. Total RNA was isolated from tissues using an RNA mini-prep kit (Tiangen, Beijing, China), and total RNA from cells was isolated using Multisource total RNA mini-prep kit (Axygen, Suzhou, China). For quantitative real-time polymerase chain reaction (qRT-PCR), RNA was reverse-transcribed to cDNA using PrimeScript RT Master Mix (Takara, Tokyo, Japan) following the manufacturer's instructions. qRT-PCR was performed by SYBR Premix EX Taq (Takara), and the sequences of primers are listed in Supplemental Table 2. RT-PCR assays were performed in Applied Biosystem Step-One Plus system with the following procedure: denaturation at $95^{\circ} \mathrm{C}$ for 30 seconds, followed by 40 cycles at $95^{\circ} \mathrm{C}$ for 5 seconds and then at $60^{\circ} \mathrm{C}$ for 30 seconds. As PPIB was stable and highly expressed in patients' tissues and glyceraldehyde-3-phosphate dehydrogenase (GAPDH) was suitable for cell analysis, results were quantified by normalization to $G A P D H$ in cell lines and PPIB in patient tissues. $2^{-\Delta \Delta \mathrm{Ct}}$ was used to calculate relative gene expression.

Western Blot and Antibodies. Tissues and cells proteins were lysed using radioimmunoprecipitation assay lysis buffer $[50 \mathrm{mM}$ Tris (pH 7.4), $150 \mathrm{mM} \mathrm{NaCl}, 1 \%$ Triton X-100, 1\% sodium deoxycholate, $0.1 \%$ SDS, sodium orthovanadate, sodium fluoride, EDTA, leupeptin; Beyotime, China]. The protein lysis was separated by centrifugation at $20,000 \mathrm{~g}$ under $4^{\circ} \mathrm{C}$ for 10 minutes, and protein concentration was quantified by BCA protein assay kit (Beyotime). Protein lysates were heated at $100^{\circ} \mathrm{C}$ for 10 minutes for denaturation with loading buffer (Takara). Protein lysates were isolated by $90 \mathrm{~V}, 30$ minutes in 5\% stacking gel, and $120 \mathrm{~V}, 70$ minutes in $15 \%$ separating gel. Proteins were then transferred to $0.45 \mu \mathrm{m}$ polyvinylidene difluoride membranes (Millipore, MA), blocked using $5 \%$ skim milk at room temperature for 2 hours, and then incubated with anti-SLC51A antibody (1:250 dilution, PAB20435; Abnova) and anti-GAPDH antibody (1:4000 dilution, KC-5G4; Kangchen), respectively. The immune complexes were detected and visualized with EZECL (4A Biotech, China) according to the manufacturer's protocol.

Chromatin Immunoprecipitation. Tissues and cells were crosslinked using 1.1\% formaldehyde (Thermo Forma); tissues and cells were incubated for 30 and 15 minutes, respectively, at room temperature, and the crosslinking state was quenched with $0.125 \mathrm{M}$ glycine. To shear the chromatin, tissues and cells were treated with $10 \mathrm{U}$ MNase $\mathrm{ml}^{-1}$ (Thermo Fisher Scientific) for 10 minutes at $37^{\circ} \mathrm{C}$, followed by tissue sonication for 30 minutes (cells for 15 minutes) at $4^{\circ} \mathrm{C}$. Sheared chromatin was incubated with $1.5 \mu \mathrm{g}$ antibody at $4^{\circ} \mathrm{C}$ overnight, and then the mixture was incubated with Protein $\mathrm{G}$ beads (Millipore) for 4-6 hours at $4^{\circ} \mathrm{C}$. The beads were then washed off, and DNA was collected for subsequent RT-PCR. The enrichment was indicated as percent of input (Lee et al., 2006). Primers used in chromatin immunoprecipitation (ChIP)-qPCR were listed in Supplemental Table 2. The antibodies used for the ChIP were as follows: anti-H3 (Ab1791; Abcam, Cambridge, MA), anti-H3Ac (06-599; Millipore), anti-H3K4me3 (ab8580; Abcam), anti-H3K4me2 (07-030; Millipore), anti-H3K9Ac (ab4441; Abcam), anti-H3K18Ac (ab1191; Abcam), anti-H3K27Ac (ab4729; Abcam), anti-H3K27me3 (07-449; Millipore), anti-H4Ac (41384; Abcam), 
anti-H3K9me3 (Active motif; 39161), and normal rabbit IgG (sc2027; Santa Cruz Biotechnology) as control. Every value of reference genes in samples were assayed, and the results of patient numbers 13,24 , and 34 were confidential and representative, owing to the good normalization of negative reference genes. These three representative results were presented.

Small Interfering RNA-Mediated Gene Knockdown. HCT116 and HT29 cells were transfected using small interfering RNA (siRNA) for HDACs and p300, which were synthesized by GenePharma (Shanghai). Transfect reagent lipofectamine 3000 (Life Technologies) was previously incubated with suitable amounts of siRNAs for 5 minutes at room temperature, and then the mixture was added into precultured cells in 60\%-80\% confluence for transient transfection. Negative control siRNA was also provided by GenePharma and added to cells as the negative control group. For HCT15, final concentration of siRNAs targeted at HDACs were $30 \mathrm{nM}$; for HT29, the final concentrations of siRNAs were $40 \mathrm{nM}$ for HDACs and $100 \mathrm{nM}$ for si-p300. After 48 hours, total RNA was extracted from cells for later analysis. The sequences of siRNAs were listed in Supplemental Table 3.

Luciferase Assay. OST $\beta$ promoter fragments were amplified with primers listed in Supplemental Table 2 and cloned into pGL3basic (Promega) for the construction of reporter plasmid. The luciferase assay was performed in HEK293 cell line. HEK293 cells were precultured to $70 \%-90 \%$ confluence in a 24 -well plate and then transfected with $O S T \beta$ promoter reporter plasmid. Cells were respectively treated with $50 \mu \mathrm{M}$ CTB and si-p300 for 48 hours to compare relative light units 1 and 2 , which indicated the firefly luciferase activity from promoter constructs normalized to the corresponding Renilla luciferase.

Statistical Analysis. Results were expressed as means \pm S.D. Paired $t$ test was used for the paired cancer and adjacent normal tissues obtained from patients. A two-tailed $t$ test was used for the statistical comparison between two experiment groups.

\section{Results}

OST $\alpha$ and OST $\beta$ Are Repressed in CRC. Data gained from the database Oncomine (Compendia Bioscience, Arm

Arbor, MI) showed that $O S T \alpha$ and $O S T \beta$ mRNA expression were distinctly downregulated in CRC tissues (Fig. 1a and b). We analyzed 93 pairs of colon cancer tissues and the $O S T \alpha$ and OST $\beta$ expression levels of pair-matched adjacent normal cancer tissues by using qRT-PCR. We found that $O S T \alpha$ and OST $\beta$ mRNA expression was obviously declined in CRC tissues compared with that in normal tissues, which was accordant with the findings from Oncomine (Fig. 2a and b). As shown in Fig. 2e, protein levels of OST $\beta$ expression also confirmed this discovery. To conclude, regardless of gender, age, location, TNM stage (T: tumor, N: regional lymph node, M: metastasis), and cancer subtype (Supplemental Figs. 1 and 2), OST $\alpha$ and OST $\beta$ transporter expression was repressed in CRC.

Reduced H3K27Ac at OST $\beta$ Promoter Region Leads to OST $\boldsymbol{\beta}$ Repression in CRC. Next, considering that epigenetics plays key roles in gene expression regulation, we attempted to confirm the epigenetic mechanism accounting for OST $\alpha$ and OST $\beta$ repression in CRC. DAC, a demethylating reagent that blocks the cellular DNA methyltransferases process (Nie et al., 2014), and TSA, an HDAC inhibitor that elevates the level of histone acetylation and promotes the transcription process, were used to treat CRC cell lines HT29 and HCT15 to see whether they would reduce OST $\alpha$ and OST $\beta$ expression (Codd et al., 2009).

After DAC treatment, neither OST $\alpha$ nor OST $\beta$ mRNA expression were elevated (Supplemental Fig. 3b; Supplemental Fig. 4a), so we speculated that the DNA methylation state was not the main regulator of OST $\alpha$-OST $\beta$ repression in CRC. However, after TSA treatment, we found OST $\beta$ transcription was positively related to TSA dosage in HT29 and HCT115 (Fig. 3a and b), and the same results were discovered in protein levels (Supplemental Fig. 3c). But for OST $\alpha$, expression of mRNA was slightly influenced after TSA treatment (Supplemental Fig. 3a), which was also parallel with ChIP-qPCR results

B
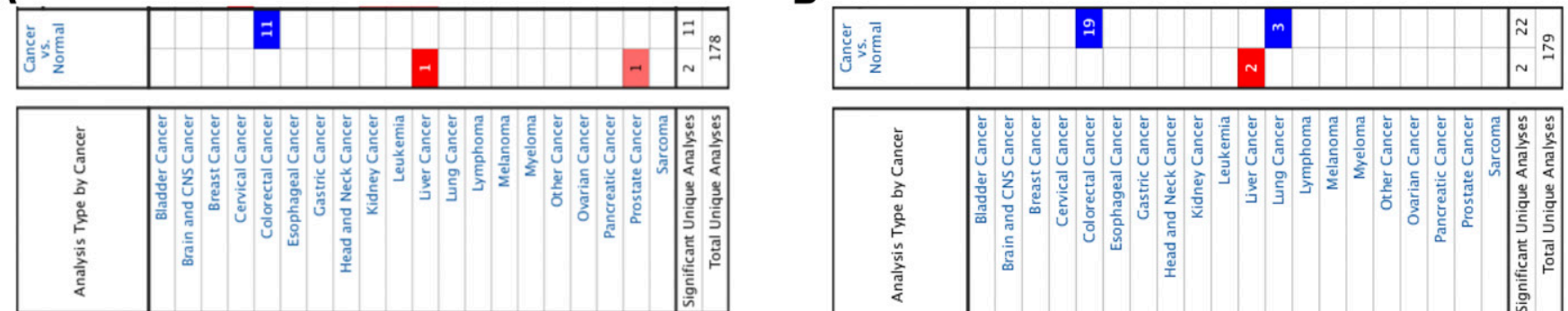

C

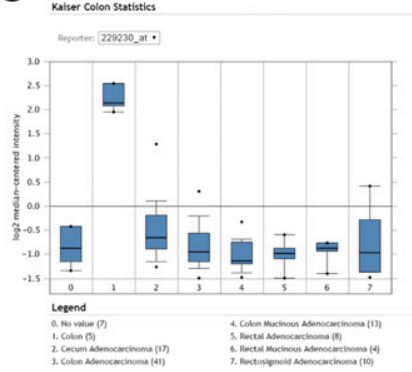

D

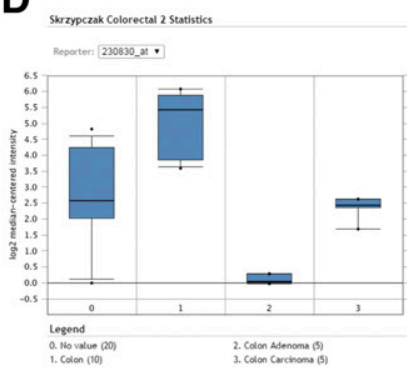

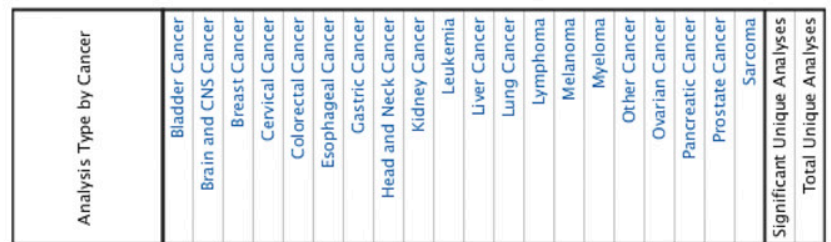

$\mathbf{E}$

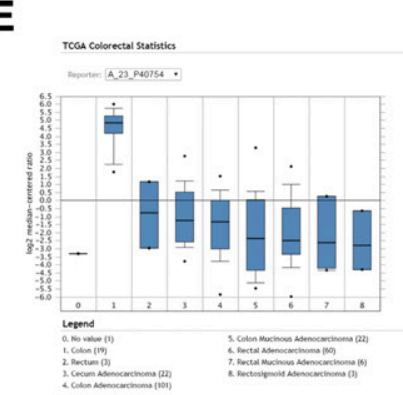

$\mathbf{F}$

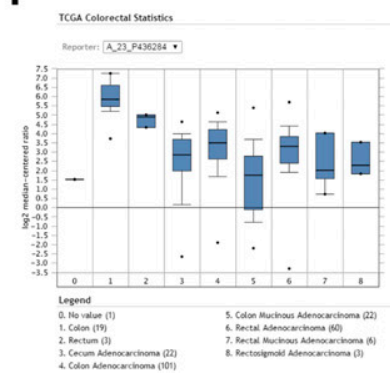

Fig. 1. (A and B) Summary of OST $\alpha$ and OST $\beta$ expression in various human cancers compared with normal tissues in Oncomine. Blue: downregulated in cancer; red: upregulated in cancer. Cell color is determined by the best gene rank percentile for the analyses within the cell. (C and D) OST $\beta$ expression in Kaiser Colon Statistics and Skrzypczak Colorectal 2 Statistics. (E and F) OST $\alpha$ and OST $\beta$ expression in TCGA Colorectal statistics. 
A

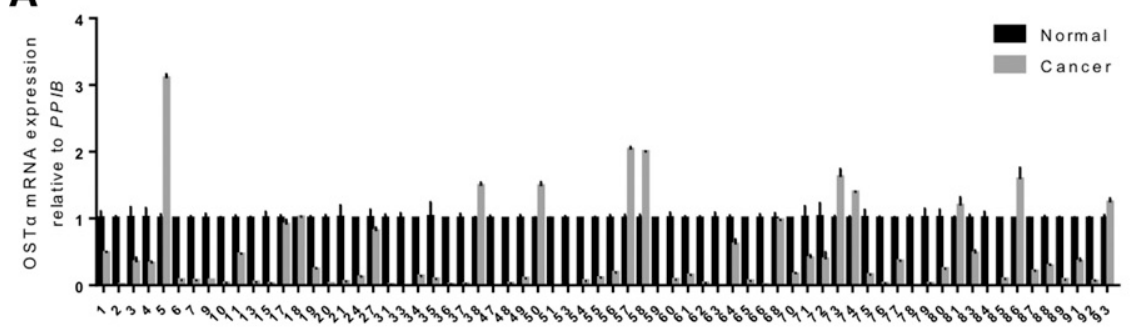

C

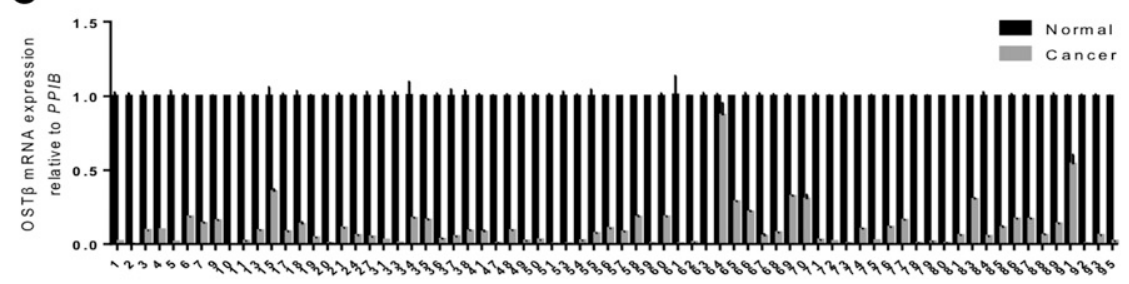

E

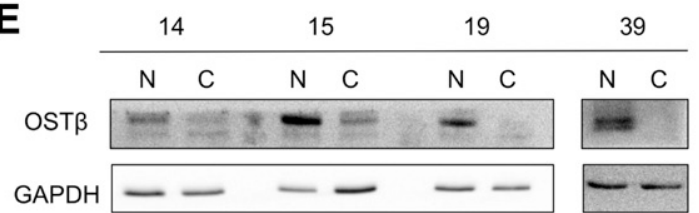

B
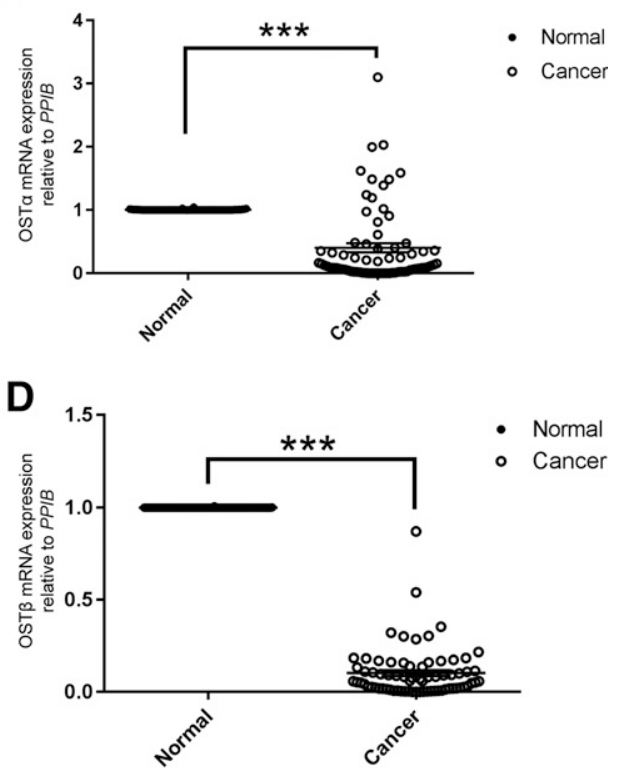

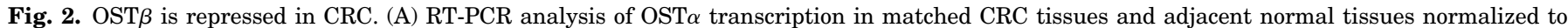

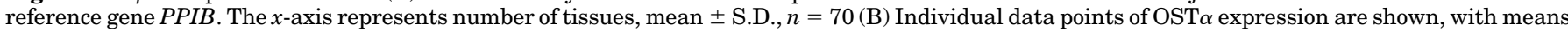

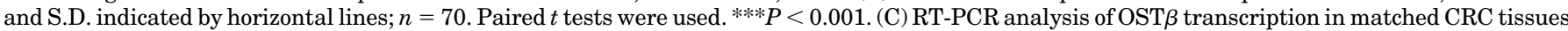

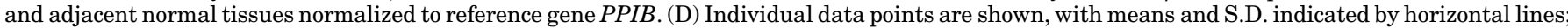

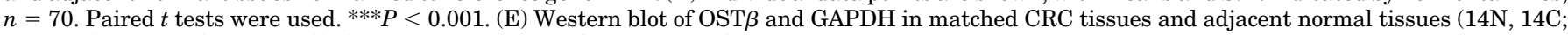
$15 \mathrm{~N}, 15 \mathrm{C} ; 19 \mathrm{~N}, 19 \mathrm{C} ; 39 \mathrm{~N}, 39 \mathrm{C}$ ). C, cancer tissues; N, adjacent normal tissues.

(Supplemental Fig. 3c). Collectively, DNA methylation and histone acetylation may not be responsible for OST $\alpha$ repression in CRC.

To verify whether histone acetylation repressed OST $\beta$ expression in CRC, we performed ChIP-qPCR experiments. HT29 and HCT15 cells were cultured in medium with $1 \mu \mathrm{M}$ TSA for 24 hours. Then, in both cells, we found the H3K27Ac level at OST $\beta$ promoter region was upregulated whereas H4Ac was downregulated after TSA treatment (Fig. 3d and e). As we know, TSA was intended to inhibit HDAC and promote the histone acetylation. The result indicated that TSA could upregulate the expression of H3K27Ac as well as the mRNA expression of OST $\beta$, whereas H4Ac state was not consistent with this tendency. In that case, we speculated that H3K27Ac played more important roles compared with H4Ac. To test this hypothesis, we also did ChIP-qPCR experiments with CRC tissues, and the results were in accordance with cells (Fig. 3f and g). We found that CRC tissues presented consistently a lower H3K27Ac state at $O S T \beta$ promoter region than adjacent normal tissues. However, the H4Ac state at OST $\beta$ promoter region varied without rules. This data may provide more evidence that indicates that H3K27Ac plays a dominant role in modulating OST $\beta$ expression in CRC.

We designed two different pairs of primers for all ChIPqPCR analysis mentioned above to cover the whole $2000 \mathrm{bp}$ of the the OST $\beta$ promoter region from an overall perspective rather than only focus on a specific range in the promoter. Both primers presented similar results, which indicated that all parts of the promoter region were intended to be acetylated.
The RT-PCR results for both pairs of primers are shown in Fig. 3 and Supplemental Fig. 4. Taken together, these results revealed that lower H3K27Ac state at OST $\beta$ promoter region leads to OST $\beta$ repression in CRC.

Downregulation of p300 in CRC Causes OST $\beta$ Repression in CRC. Previous reports have proved that histone acetylation state is influenced by enzymes, such as HATs and HDACs, and TSA was reported to inhibit Zincdependent class I and class II histone deacetylases, including HDAC1-10 (Seto and Yoshida, 2014). Therefore, ChIP evaluation was performed in CRC tissues to find out which specific HAT or HDAC was responsible for OST $\beta$ repression during CRC development.

We found that HDAC2 and HDAC7 were upregulated in CRC tissues compared with adjacent normal tissues, and HDAC9 was downregulated (Supplemental Fig. 5). It seemed HDAC2 and HDAC7 might be the main factors to influence OST $\beta$ expression; however, further siRNA knockdown assay did not support this speculation. Expression of $O S T \beta$ was not accordingly elevated after cells were transfected with siRNAs targeted at corresponding HDACS (Supplemental Figs. 6 and 7). Hence, it can be concluded that HDACs might not be the dominant factors responsible for OST $\beta$ repression in CRC.

Then, we began research on HATs to further test which HATs participated in modulating OST $\beta$ expression. Unlike HDACs, HATs modify specific sites on histones. Combining the previous research in our laboratory on HATs (Ye et al., 2018), we found p300 modified H3K27Ac and H3K18Ac in vivo (Holmqvist and Mannervik, 2013). Interestingly, mRNA expression of $p 300$ was 
A

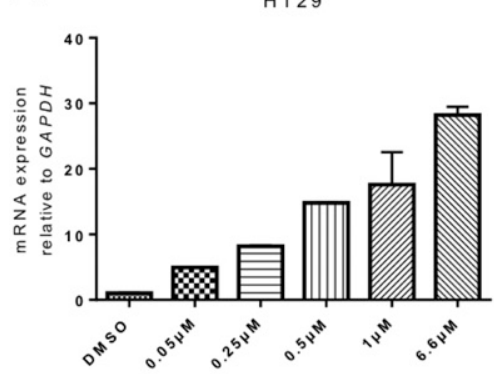

D

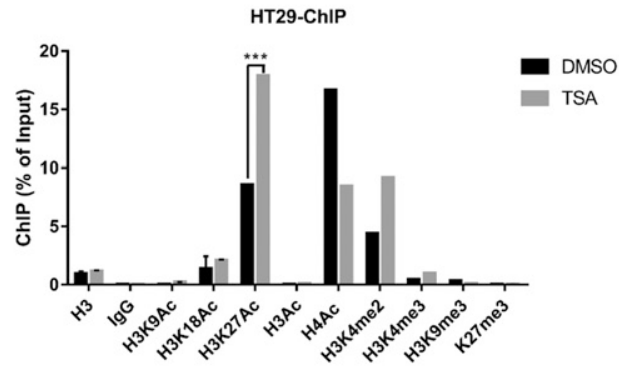

F

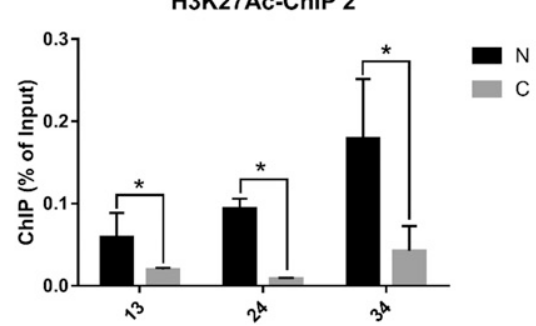

B

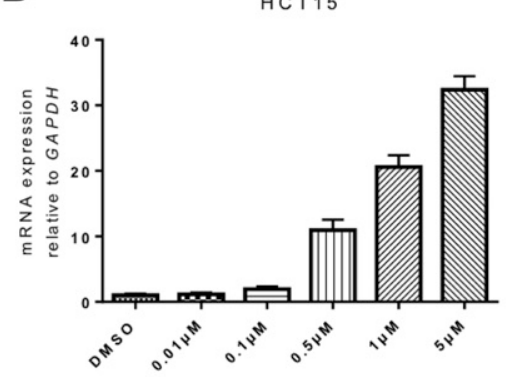

$E$

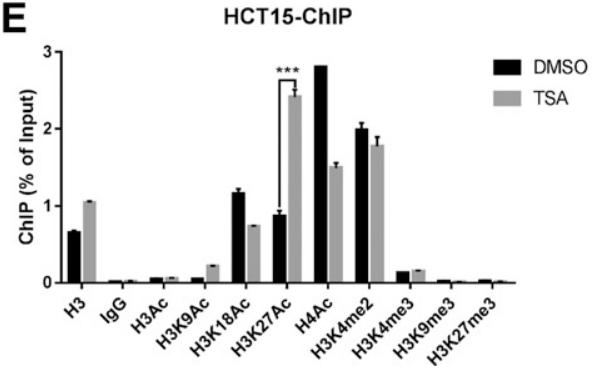

G

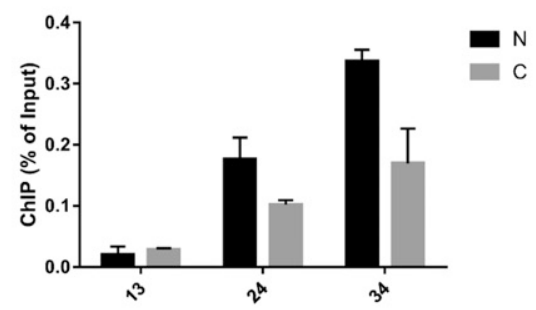

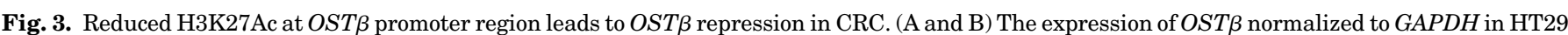

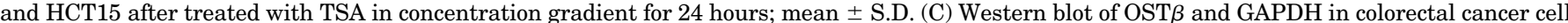

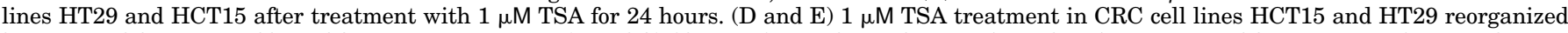

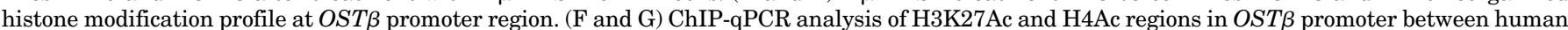

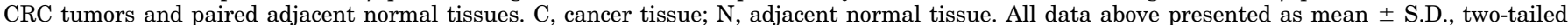
unpaired $t$ test. $* P<0.05 ; * * * P<0.001$.

demonstrated to be downregulated in CRC tissues as predicted (Fig. 4a). In vitro experiments were all performed in CRC cell line HT29, and because OST $\beta$ expression in HCT15 was relatively low, it was difficult to detect its expression accurately. We then treated HT29 cells with p300 activator CTB (Mantelingu et al., 2007; Furdas et al., 2012; Dastjerdi et al., 2013) and inhibitor C646 (Bowers et al., 2010; Gaddis et al., 2015). We found that when p300 was activated, OST $\beta$ expression was elevated as well (Fig. 4b); when p300 was inhibited, OST $\beta$ expression also decreased dramatically (Fig. 4c). Moreover, to further confirm the association between p300 and OST $\beta$ expression, we also designed siRNA for p300 to observe variation on OST $\beta$ expression after $p 300$ is degraded. As predicted, after p300 knockdown, OST $\beta$ expression was also downregulated (Fig. 4d).

We had already proved H3K27Ac state-controlled OST $\beta$ expression in CRC, so we wanted to verify if p300 focused on regulating H3K27Ac at OST $\beta$ promoter region to control its expression. ChIP-qPCR results were performed to support this speculation. Results show that after being treated with p300 activator, H3K27Ac state was enhanced at OST $\beta$ promoter region; conversely, inhibiting p300 decreased H3K27Ac state (Fig. 5a-d). Results of luciferase assay also showed similar outcomes, which were that CTB promoted OST $\beta$ promoter activity and si-p300 downregulated its activity (Fig. 5e and f). In conclusion, p300 regulates H3K27Ac level at OST $\beta$ promoter region to control its expression in CRC.

\section{Discussion}

The results of recent studies have confirmed that bile acid accumulation in vivo could promote the tumorigenesis of colorectal cancer and trigger the colonic mucosa with undesirable outcomes, such as DNA damage, oxidative/nitrosative stress, and apoptosis (Ajouz et al., 2014; Gadaleta et al., 2017). Primary bile acid was synthesized from cholesterol catabolism in liver and absorbed in distal small intestine, where they are transformed into secondary bile acids by bacteria in the intestine. Bile acids are efficiently reabsorbed via the portal venous circulation in the ileum and continue to go through cycles between the liver and intestine (Ballatori et al., 2009).

It can be seen that the accumulation of bile acid was closely associated with various bile acid transporters. Raufman et al. (2015) found that mice deficient in one specific bile acid transporter (ASBT, ileal apical sodium-dependent bile acid transporter) were more likely to induce colon tumor formation and formed larger colon adenocarcinomas after treatment because of a lack of bile acid uptake in the distal small 
A

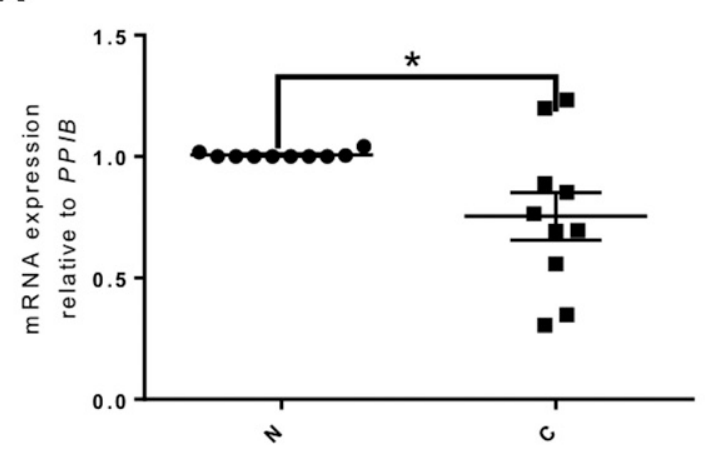

C

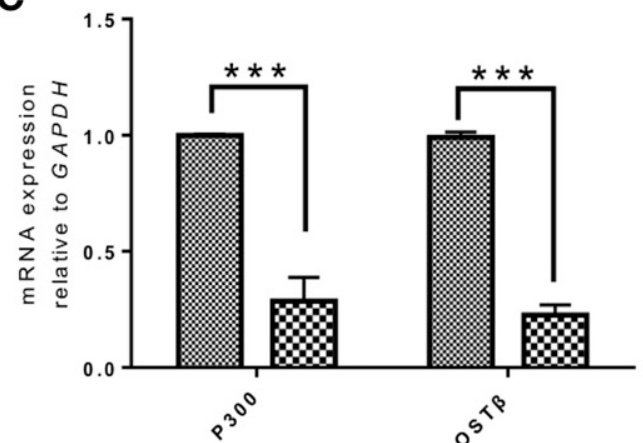

B

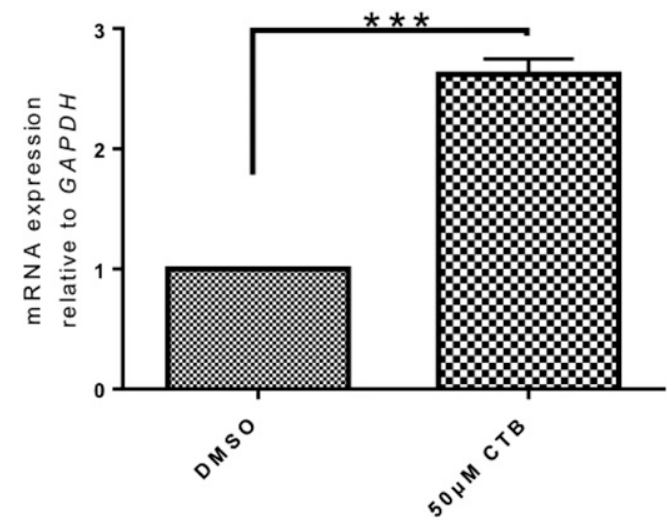

D

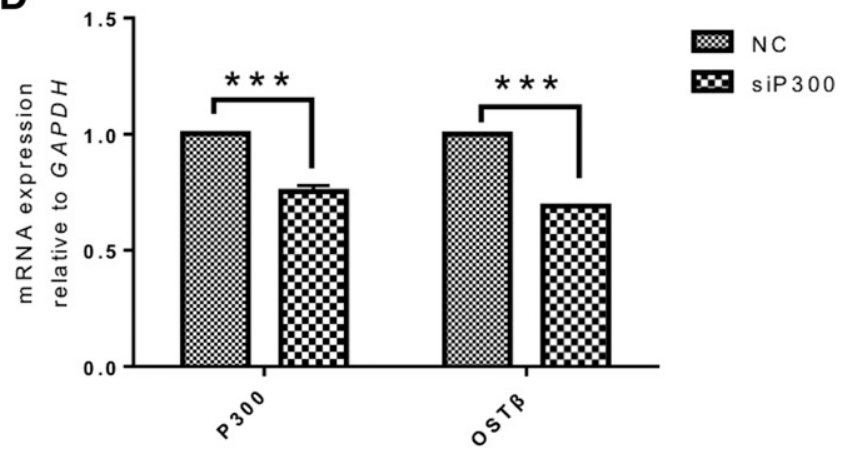

Fig. 4. P300 regulates $O S T \beta$ expression. (A) RT-PCR analysis of $p 300$ transcription in matched CRC tissues and adjacent normal tissues normalized to reference gene PPIB ( $n=10$, two-tailed paired $t$ test). Lines represent mean with S.D. C cancer tissue; N, adjacent normal tissue. (B) The mRNA expression of OST $\beta$ normalized to GAPDH in HT29 after treatment with $50 \mu \mathrm{M}$ CTB for 48 hours. (C) The mRNA expression of OST $\beta$ normalized to

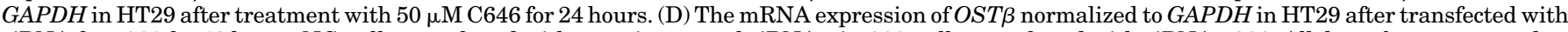
siRNA for $p 300$ for 48 hours. NC, cells transfected with negative control siRNA; si-p300, cells transfected with siRNA-p300. All data above presented as mean \pm S.D., two-tailed unpaired $t$ test. $* P<0.05 ; * * P<0.01 ; * * P<0.001$.

intestine. We found the expression of efflux bile acid transporters OST $\alpha$-OST $\beta$ was noticeably reduced in colorectal cancer tissues compared with control, and this result was demonstrated by Oncomine Cancer Transcriptome database and Western blot RT-PCR assay. OST $\alpha$-OST $\beta$ complex is a bile acid transporter first identified in 2001 (Wang et al., 2001). So far, there have been no studies that have clarified the direct interaction between OST $\alpha-\beta$ transporters and colon rectal cancer development. Except for the common list substrates of OST $\alpha$-OST $\beta$ transporters, nonbile acids such as estrone-3sulfate, digoxin, prostaglandin $\mathrm{E} 2$, and dehydroepiandrosterone-3-sulfate were also the substrates for OST $\alpha-\beta$ (Ballatori et al., 2009; (Dawson et al., 2009); Klaassen and Aleksunes, 2010 ). In this study, we mainly focused on the OST $\alpha-\beta$ transporters localized in the intestine, and the dominant substrates of OST $\alpha-\beta$ in the intestine are bile acids. As for OST $\alpha-\beta$ transporters localized in other organs such as brain or adrenal gland, they could modulate steroid homeostasis, contributing to neurosteroid action (Fang et al., 2010), and those localized in kidney or liver are responsible for conjugated steroids traffic (Ballatori et al., 2008). The function of intestinal OST $\alpha-\beta$ transporters in modulating bile acid reabsorption and its relationship with colon cancer carcinogenesis was the main focus in this article. Their regulation mechanism was rarely uncovered, especially in the process of tumorigenesis.

We continue to investigate the potential epigenetics mechanism of these two subunits of the transporter in colorectal cancer. Actually, studies have proven that epigenetics alterations play a vital role in regulating colorectal cancer development and at the same time are considered as biomarkers for CRC therapeutic applications (Okugawa et al., 2015). For example, in our laboratory's previous study, TSA induced the expression of concentrative nucleoside transporter 2 through reducing the level of deacetylation in the promoter region of concentrative nucleoside transporter 2 gene as well as reversed drug resistance in colorectal cancer therapy (Ye et al., 2018). DNA methylation inhibitor decitabine and histone deacetylase inhibitor vorinostat could noticeably evaluate the expression of human organic cation transporter OCT2 (Organic cation transporter 2)in renal cell carcinoma (Zhu et al., 2019), and the abnormal expression of OCT2 caused resistance to anticarcinogen-like oxaliplatin in renal cell carcinoma, bringing difficulties to cancer therapy (Chen et al., 2019). Furthermore, silencing of other family members of histone acetyltransferases, such as MOF ( a member of the Moz-Ybf2/Sas3-Sas2-Tip60 family of HATs), causes genomic instability, suppressing of gene transcription, and promotion of different tumorigenesis, including colorectal cancer (Su et al., 2016). Generally, the histone acetylation level was positively correlated with transcriptional activity of genes. Our study found that not only DNA methylation but also histone acetylation levels of the $O S T \beta$ promoter region were evaluated after treated with DNA transmethylase (by DAC) and HDAC (by TSA), respectively, and we finally concluded 
A

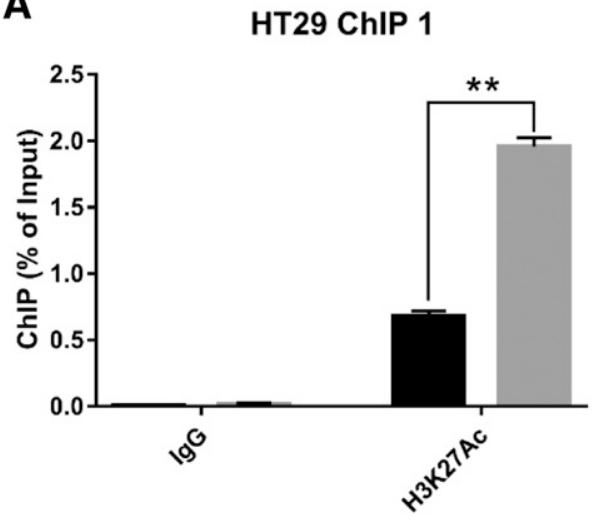

C

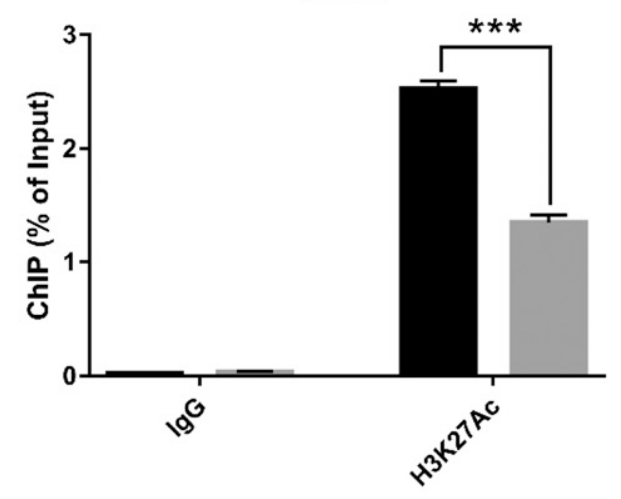

E

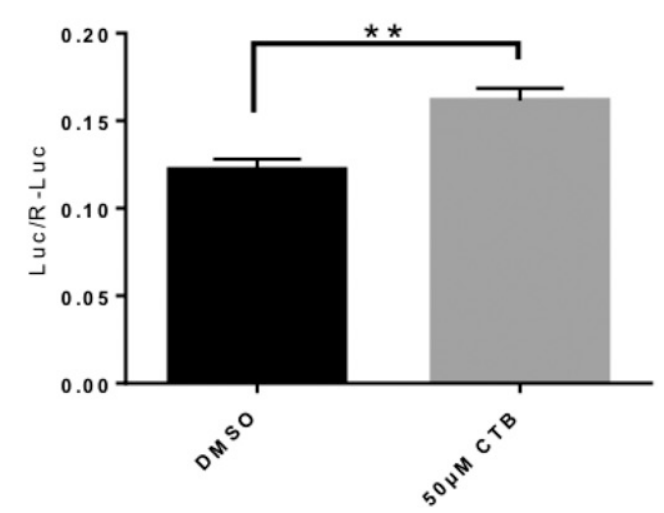

B

DMSO

$50 \mu \mathrm{M}$ CTB

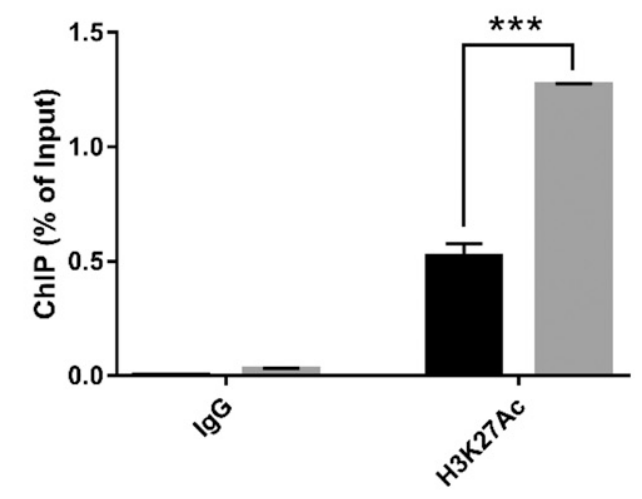

HT29

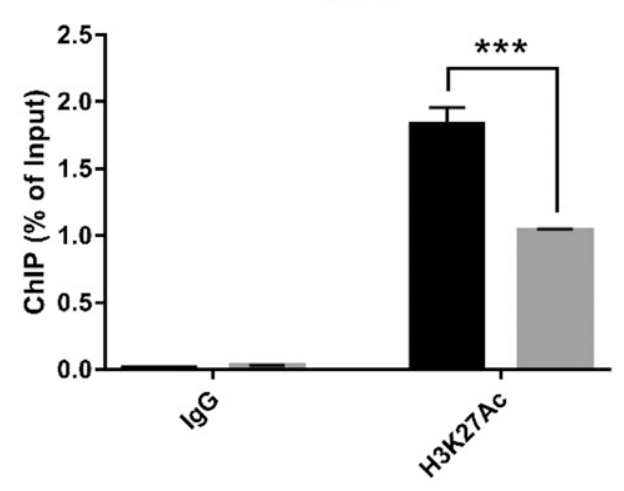

F

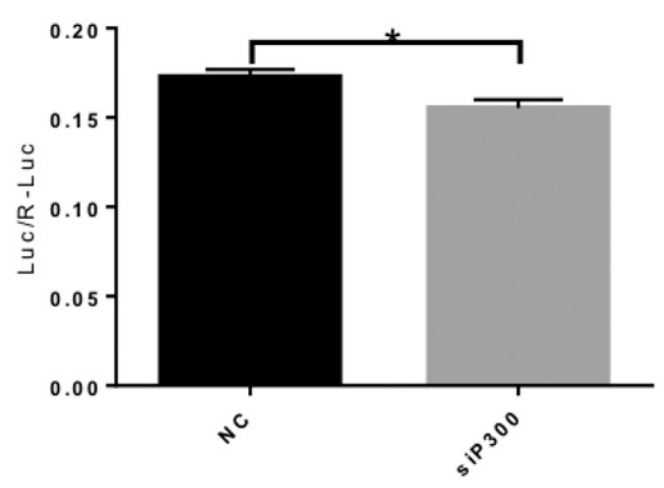

DMSO

$50 \mu \mathrm{M}$ СТВ

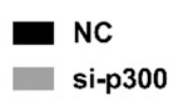

si-p300

Fig. 5. P300 regulates H3K27Ac state in OST $\beta$ promoter region. (A and B) $50 \mu \mathrm{M}$ CTB treatment for 48 hours in CRC cell line HT29 upregulated H3K27Ac at OST $\beta$ promoter region. (C and D) P300-siRNA treatment for 48 hours in CRC cell line HT29 downregulated H3K27Ac at OST $\beta$ promoter region. NC, cells transfected with negative controls; si-p300, cells transfected with si-p300. (E and F) Dual-luciferase reporter gene assay in HEK293 cells. All data above presented as mean \pm S.D., two-tailed unpaired $t$ test. $* P<0.05 ; * * P<0.01 ; * * P<0.001$.

that histone acetylation was the dominant reason responsible for repressing OST $\beta$ expression. By ChIP assay, we discovered that the downregulation of OST $\beta$ transcription was mediated by acetylation level of H3K27Ac site. In colorectal cancer tissues, the acetylation level was also reduced compared with normal adjacent tissues.

Based on the understanding of the epigenetics regulation of OST $\beta$ transcription, we continued to discover the potential factors within HATs and HDACs to regulate H3K27Ac state. From the previous study, p300 is a main regulator of modulating H3K27Ac state and a well-studied HAT ( Raisner et al., 2018; Wong et al., 2018). Combined with the qRT-PCR, drug treatment, siRNA knockdown, and luciferase assay, we could finally draw the conclusion that p300 regulates H3K27Ac at OST $\beta$ promoter region to control its expression in CRC.

To our knowledge, our study first revealed the epigenetics regulation mechanism of $\mathrm{OST} \beta$ in colorectal cancer. This finding could be clinically meaningful because OST $\beta$ dysregulation may be related to higher concentrations of DCA and LCA, which have already been proved to be carcinogenic factors, in the colorectal region. Taken together, our study indicated that the reduced expression of p300 caused H3K27 deacetylation in OST $\beta$ promoter region and hence led to the downregulated expression of OST $\beta$ in CRC to mediate the export of bile acid. 
This result gives new insight into the epigenetic effects toward the main factors in colorectal cancer and suggests that in the future, cancer therapy could focus on targeting epigenetic transcriptional factors, such as p300, to treat cancer.

\section{Authorship Contributions}

Participated in research design: Zhou, C. Ye, Zeng, Yu.

Conducted experiments: Zhou, C. Ye, Lou, Chen, Lei, Guo.

Contributed new reagents or analytic tools: C. Ye, Liu, S. Ye.

Performed data analysis: Zhou, Lou, Zeng, Yu.

Wrote or contributed to the writing of the manuscript: Zhou, C. Ye, Yu.

\section{References}

Ajouz H, Mukherji D, and Shamseddine A (2014) Secondary bile acids: an underrecognized cause of colon cancer. World $J$ Surg Oncol 12:164.

Ballatori N, Christian WV, Lee JY, Dawson PA, Soroka CJ, Boyer JL, Madejczyk MS, and Li N (2005) OSTalpha-OSTbeta: a major basolateral bile acid and steroid transporter in human intestinal, renal, and biliary epithelia. Hepatology 42 $1270-1279$.

Ballatori N, Christian WV, Wheeler SG, and Hammond CL (2013) The heteromeric organic solute transporter, OST $\alpha$-OST $\beta /$ SLC51: a transporter for steroid-derived molecules. Mol Aspects Med 34:683-692.

Ballatori N, Fang F, Christian WV, Li N, and Hammond CL (2008) Ostalpha-Ostbeta is required for bile acid and conjugated steroid disposition in the intestine, kidney, and liver. Am J Physiol Gastrointest Liver Physiol 295:G179-G186.

Ballatori N, Li N, Fang F, Boyer JL, Christian WV, and Hammond CL (2009) OST alpha-OST beta: a key membrane transporter of bile acids and conjugated steroids. Front Biosci 14:2829-2844.

Bernstein C, Holubec H, Bhattacharyya AK, Nguyen H, Payne CM, Zaitlin B, and Bernstein $\mathrm{H}$ (2011) Carcinogenicity of deoxycholate, a secondary bile acid. Arch Toxicol 85:863-871.

Bird A (2002) DNA methylation patterns and epigenetic memory. Genes Dev 16:6-21. Bowers EM, Yan G, Mukherjee C, Orry A, Wang L, Holbert MA, Crump NT, Hazzalin CA, Liszczak G, Yuan H, et al. (2010) Virtual ligand screening of the p300/CBP histone acetyltransferase: identification of a selective small molecule inhibitor. Chem Biol 17:471-482.

Brenner H, Kloor M, and Pox CP (2014) Colorectal cancer. Lancet 383:1490-1502.

Center MM, Jemal A, Smith RA, and Ward E (2009) Worldwide variations in colorectal cancer. CA Cancer J Clin 59:366-378.

Chen L, Chen L, Qin Z, Lei J, Ye S, Zeng K, Wang H, Ying M, Gao J, Zeng S, et al. (2019) Upregulation of miR-489-3p and miR-630 inhibits oxaliplatin uptake in renal cell carcinoma by targeting OCT2. Acta Pharm Sin B 9:1008-1020.

Chiang JY (2009) Bile acids: regulation of synthesis. J Lipid Res 50:1955-1966.

Christian WV, Li N, Hinkle PM, and Ballatori N (2012) $\beta$-Subunit of the Ost $\alpha$-Ost $\beta$ organic solute transporter is required not only for heterodimerization and trafficking but also for function. $J$ Biol Chem 287:21233-21243.

Codd R, Braich N, Liu J, Soe CZ, and Pakchung AA (2009) Zn(II)-dependent histone deacetylase inhibitors: suberoylanilide hydroxamic acid and trichostatin A. Int J Biochem Cell Biol 41:736-739.

Dastjerdi MN, Salahshoor MR, Mardani M, Hashemibeni B, and Roshankhah S (2013) The effect of CTB on P53 protein acetylation and consequence apoptosis on MCF-7 and MRC-5 cell lines. Adv Biomed Res 2:24.

Dawson PA, Lan T, and Rao A (2009) Bile acid transporters. J Lipid Res 50: 2340-2357.

Fang F, Christian WV, Gorman SG, Cui M, Huang J, Tieu K, and Ballatori N (2010) Neurosteroid transport by the organic solute transporter OST $\alpha$-OST $\beta$. J Neurochem 115:220-233.

Farhana L, Nangia-Makker P, Arbit E, Shango K, Sarkar S, Mahmud H, Hadden T, $\mathrm{Yu}$ Y, and Majumdar AP (2016) Bile acid: a potential inducer of colon cancer stem cells. Stem Cell Res Ther 7:181.

Favoriti P, Carbone G, Greco M, Pirozzi F, Pirozzi RE, and Corcione F (2016) Worldwide burden of colorectal cancer: a review. Updates Surg 68:7-11.
Furdas SD, Kannan S, Sippl W, and Jung M (2012) Small molecule inhibitors of histone acetyltransferases as epigenetic tools and drug candidates. Arch Pharm Weinheim) 345:7-21.

Gadaleta RM, Garcia-Irigoyen O, and Moschetta A (2017) Bile acids and colon cancer: is FXR the solution of the conundrum? Mol Aspects Med 56:66-74.

Gaddis M, Gerrard D, Frietze S, and Farnham PJ (2015) Altering cancer transcriptomes using epigenomic inhibitors. Epigenetics Chromatin 8:9.

Holmqvist PH and Mannervik M (2013) Genomic occupancy of the transcriptional coactivators p300 and CBP. Transcription 4:18-23.

Klaassen CD and Aleksunes LM (2010) Xenobiotic, bile acid, and cholesterol transporters: function and regulation. Pharmacol Rev 62:1-96.

Lee TI, Johnstone SE, and Young RA (2006) Chromatin immunoprecipitation and microarray-based analysis of protein location. Nat Protoc 1:729-748.

Liu Y, Zheng X, Yu Q, Wang H, Tan F, Zhu Q, Yuan L, Jiang H, Yu L, and Zeng S (2016) Epigenetic activation of the drug transporter OCT2 sensitizes renal cell carcinoma to oxaliplatin. Sci Transl Med 8:348ra97.

Mantelingu K, Kishore AH, Balasubramanyam K, Kumar GV, Altaf M, Swamy SN, Selvi R, Das C, Narayana C, Rangappa KS, et al. (2007) Activation of p300 histone acetyltransferase by small molecules altering enzyme structure: probed by surfaceenhanced Raman spectroscopy. J Phys Chem B 111:4527-4534.

Minucci S and Pelicci PG (2006) Histone deacetylase inhibitors and the promise of epigenetic (and more) treatments for cancer. Nat Rev Cancer 6:38-51.

Narisawa T, Magadia NE, Weisburger JH, and Wynder EL (1974) Promoting effect of bile acids on colon carcinogenesis after intrarectal instillation of N-methyl-N'-nitroN-nitrosoguanidine in rats. J Natl Cancer Inst 53:1093-1097.

Nie J, Liu L, Li X, and Han W (2014) Decitabine, a new star in epigenetic therapy: the clinical application and biological mechanism in solid tumors. Cancer Lett 354:12-20.

Okugawa Y, Grady WM, and Goel A (2015) Epigenetic alterations in colorecta cancer: emerging biomarkers. Gastroenterology 149:1204-1225.e12.

Plotnikoff GA (2014) Three measurable and modifiable enteric microbial biotransformations relevant to cancer prevention and treatment. Glob Adv Health Med 3:33-43.

Raisner R, Kharbanda S, Jin L, Jeng E, Chan E, Merchant M, Haverty PM, Bainer R, Cheung T, Arnott D, et al. (2018) Enhancer activity requires CBP/P300 bromodomaindependent histone H3K27 acetylation. Cell Rep 24:1722-1729.

Raufman JP, Dawson PA, Rao A, Drachenberg CB, Heath J, Shang AC, Hu S, Zhan M, Polli JE, and Cheng K (2015) Slc10a2-null mice uncover colon cancer-promoting actions of endogenous fecal bile acids. Carcinogenesis 36:1193-1200.

Seto E and Yoshida M (2014) Erasers of histone acetylation: the histone deacetylase enzymes. Cold Spring Harb Perspect Biol 6:a018713.

Seward DJ, Koh AS, Boyer JL, and Ballatori N (2003) Functional complementation between a novel mammalian polygenic transport complex and an evolutionarily ancient organic solute transporter, OSTalpha-OSTbeta. J Biol Chem 278:27473-27482

Su J, Wang F, Cai Y, and Jin J (2016) The functional analysis of histone acetyltransferase MOF in tumorigenesis. Int J Mol Sci 17:99.

Wang W, Seward DJ, Li L, Boyer JL, and Ballatori N (2001) Expression cloning of two genes that together mediate organic solute and steroid transport in the liver of a marine vertebrate. Proc Natl Acad Sci USA 98:9431-9436.

Wang Z, Zang C, Cui K, Schones DE, Barski A, Peng W, and Zhao K (2009) Genomewide mapping of HATs and HDACs reveals distinct functions in active and inactive genes. Cell 138:1019-1031.

Wong CK, Wade-Vallance AK, Luciani DS, Brindle PK, Lynn FC, and Gibson WT (2018) The p300 and CBP transcriptional coactivators are required for $\beta$-cell and $\alpha$-cell proliferation. Diabetes 67:412-422.

Ye C, Han K, Lei J, Zeng K, Zeng S, Ju H, and Yu L (2018) Inhibition of histone deacetylase 7 reverses concentrative nucleoside transporter 2 repression in colorectal cancer by up-regulating histone acetylation state. Br J Pharmacol 175:4209-4217.

Yin J, Sun W, Li F, Hong J, Li X, Zhou Y, Lu Y, Liu M, Zhang X, Chen N, et al. (2020) VARIDT 1.0: variability of drug transporter database. Nucleic Acids Res 48: D1042-D1050.

Zhu Q, Yu L, Qin Z, Chen L, Hu H, Zheng X, and Zeng S (2019) Regulation of OCT2 transcriptional repression by histone acetylation in renal cell carcinoma. Epigenetics 14:791-803.

Address correspondence to: Lushan Yu, Institute of Drug Metabolism and Pharmaceutical Analysis, College of Pharmaceutical Sciences, Zhejiang University, Hangzhou, Zhejiang, China. E-mail: yuls@zju.edu.cn 